\title{
Sex Differences in Autoimmune Multimorbidity in Type 1 Diabetes Mellitus and the Risk of Cardiovascular and Renal Disease: A Longitudinal Study in the United States, 2001-2017
}

\author{
Mary A.M. Rogers, PhD, MS, ${ }^{1,2}$ Melissa Y. Wei, MD, MPH, MS,,2 \\ Catherine Kim, MD, MPH, ${ }^{1,2}$ and Joyce M. Lee, MD, MPH ${ }^{2,3}$
}

\begin{abstract}
Background: Autoimmune diseases are usually more prevalent in women. The risks of cardiovascular and renal disease in those with multiple autoimmune diseases have not been fully described.

Materials and Methods: Using a national database from a large health insurer in the United States (years 20012017) containing 75 million members, we calculated age- and sex-specific co-prevalence of 12 autoimmune disorders for individuals with type 1 diabetes. We then evaluated whether concomitant autoimmune diseases were associated with renal failure, ischemic stroke, and myocardial infarction.

Results: Of the 179,248 people diagnosed with type 1 diabetes, 1 in 4 had a concomitant autoimmune disease (27.03\%; 95\% confidence interval $[\mathrm{CI}]=26.83 \%-27.24 \%)$, with hypothyroidism, rheumatoid arthritis, and celiac disease being the most common. The prevalence of autoimmune disease was 1.9 times greater in female than male patients $(p<0.001)$. In female patients with type 1 diabetes, one in three had another autoimmune disease (35.62\%; 95\% CI=35.30\%-35.94\%) compared with one in five male patients (19.17\%; 95\% $\mathrm{CI}=18.92 \%-19.42 \%)$. The risk of renal failure, ischemic stroke, and myocardial infarction increased with a greater number of concomitant autoimmune diseases $(p<0.001$, test for trend for both female and male patients). Patients with type 1 diabetes who had multiple sclerosis or myasthenia gravis experienced an approximate threefold increase in risk of ischemic stroke (odds ratio $[\mathrm{OR}]=3.57, \mathrm{OR}=3.22$, respectively). Patients with type 1 diabetes and Addison's disease had a threefold increased risk of renal failure.

Conclusions: Patients with type 1 diabetes, particularly women, frequently have coexisting autoimmune diseases that are associated with higher rates of renal failure, ischemic stroke, and myocardial infarction. Additional study is warranted, as are preventive efforts in this high-risk population.
\end{abstract}

Keywords: sex, autoimmunity, type 1 diabetes, multimorbidity

\section{Introduction}

I N the United States, 1 in 4 noninstitutionalized older adults ( $\geq 65$ years of age) have at least 2 or more chronic medical conditions and, for younger adults (18-44 years), it is 1 in $20 .{ }^{1}$ Long-term multimorbidity (i.e., multiple coexisting chronic conditions) is associated with decreased healthrelated quality of life and increased physical and cognitive impairment, disability, and mortality even among young and middle-aged adults. ${ }^{2-4}$ The burden of a lifetime spent with the daily challenges of coping with multimorbidity is particularly poignant for those who develop chronic diseases at an early age. Type 1 diabetes mellitus is one such disease, the incidence of which is greatest in children. ${ }^{5}$ Rates of type 1 diabetes, an autoimmune disease, often cluster with other autoimmune diseases such as celiac disease and thyroid disorders. $^{6-9}$ In the Type 1 Diabetes Registry, $34 \%$ of female and $19 \%$ of male patients with type 1 diabetes had additional autoimmune diseases. ${ }^{6}$ In a study of adults with type 1 diabetes using a nationwide patient database, $30 \%$ of women and

\footnotetext{
${ }^{1}$ Department of Internal Medicine, University of Michigan, Ann Arbor, Michigan.

${ }^{2}$ The Institute for Healthcare Policy and Innovation, University of Michigan, Ann Arbor, Michigan.

${ }^{3}$ Pediatric Endocrinology, Child Health Evaluation and Research Unit (CHEAR), University of Michigan, Ann Arbor, Michigan.
} 
$15.8 \%$ of men had an additional autoimmune disease, with thyroid disease being the most common. ${ }^{7}$ In a pediatric study, one-third of children with type 1 diabetes had autoantibodies for common autoimmune disorders and $6 \%$ were diagnosed with another autoimmune disease within several months after the onset of type 1 diabetes. ${ }^{9}$

Although both microvascular and macrovascular complications of type 1 diabetes have been described, ${ }^{10,11}$ population-based studies of the influence of multiple autoimmune diseases on subsequent cardiovascular and renal disease have not been fully investigated. Because the United States has the largest population with type 1 diabetes, ${ }^{12}$ we used a nationwide sample to investigate the co-prevalence of autoimmune diseases. We were particularly interested in the ages when such autoimmune diseases manifested and sex differences in prevalence. Although autoimmune diseases, in general, tend to be more common in female than male patients, type 1 diabetes is unusual in that it is more common in male than female patients, particularly after puberty. ${ }^{13}$ With this national database, we answered the following questions: (1) for a patient with type 1 diabetes of a given age and sex, what is the likelihood of concomitant autoimmune disease? and (2) are persons with multiple autoimmune disorders more likely to have severe morbidities such as renal failure, myocardial infarction, and ischemic stroke?

\section{Materials and Methods}

\section{Data source}

The data source was an integrated relational database from a nationwide health insurer serving all 50 states in the United States. Longitudinal data were extracted from January 1, 2001 through June 30, 2017 from the Clinformatics ${ }^{\circledR}$ Data Mart Database (available through OptumInsight, Eden Prairie, MN). This nationwide database contains integrated health information on $\sim 75$ million American infants, children, and adults with private health insurance, encompassing demographic and membership information, diagnoses and procedures, outpatient and inpatient services, pharmacy records, and laboratory data.

\section{Subjects}

Data were extracted on patients with type 1 diabetes using a validated algorithm with the ratio of type 1 to type 1 and type 2 diabetes diagnoses set at $\geq 0.6$. In previous analyses, this yielded a positive predictive value of $98.7 \%$ for individuals $\geq 10$ years of age (slightly more accurate than setting the ratio at $\geq 0.5$ ) and of $98.7 \%$ for those $<10$ years of age. ${ }^{14}$ An additional criterion was the use of insulin; because all patients with type 1 diabetes must use insulin to live, those patients who never used insulin were excluded. ${ }^{15}$ No restrictions were made regarding age or sex. There were $0.02 \%$ $(n=35)$ values missing for sex and these individuals were excluded.

The 12 most common autoimmune diseases in those with type 1 diabetes in the database were chosen for greater review. These were hypothyroidism (including Hashimoto's disease), rheumatoid arthritis, celiac disease, psoriasis, Grave's disease, inflammatory bowel disease, pernicious anemia, Addison's disease, systemic lupus erythematosus, multiple sclerosis, Sjögren's syndrome, and myasthenia gravis. Information regarding autoimmune diseases was extracted using previously defined diagnostic codes from both outpatient and inpatient files. ${ }^{16}$ For hypothyroidism, we excluded those with postsurgical, postablative, iodine, or iatrogenic hypothyroidism.

\section{Statistical analyses}

Initially, the age- and sex-specific prevalence of concomitant autoimmune disease in those with type 1 diabetes was calculated. The numerator was the number of patients with type 1 diabetes who had evidence of the autoimmune disease diagnoses from inpatient and/or outpatient records. The denominator was the number of patients with type 1 diabetes. Results were stratified by sex and 5-year age groups. For example, for women with type 1 diabetes who were 35-39 years of age, what percentage had rheumatoid arthritis? This prevalence was calculated for each age-sex group and reflects the likelihood of diagnosis and treatment during that time period. Of importance, the age- and sex-specific prevalence does not reflect the prevalence of autoimmune disease throughout the entire lifespan of the individual; rather, it reflects the diagnosis and treatment of that disease during a specific age period. Exact binomial $95 \%$ confidence intervals (CIs) were calculated.

The burden of multimorbidity was explored further by examining whether renal and cardiovascular diseases differ in frequency depending upon the presence of other autoimmune diseases. Outcomes of interest were severe, potentially irreversible disease including renal failure, ischemic stroke, and myocardial infarction. Logistic regression was used, with the specific renal/cardiovascular disease as the dependent variable and the number of concurrent autoimmune diseases as the explanatory variable of interest. Results were stratified by sex, and adjusted for age at the time of enrollment. Odds ratios (ORs) and 95\% CIs were calculated, as were postregression predicted probabilities for each outcome (i.e., probability that the event occurred in that particular group), which were calculated using the "margins" command after fitting the logit model. In secondary analyses, all 12 autoimmune diseases were simultaneously entered as independent variables in a logit model for each of the 3 adverse outcomes (renal failure, ischemic stroke, and myocardial infarction). Alpha was set at 0.05, two-tailed. Analyses were conducted using Stata/MP 15.1 (College Station, TX).

\section{Results}

\section{Characteristics of the study participants}

Over the entire study period (2001-2017), there were 179,248 individuals with type 1 diabetes, 85,690 of whom were female patients and 93,558 were male patients (Table 1). The mean age at enrollment was 35.2 years and the mean follow-up period was 3.4 years. Overall, one in four individuals with type 1 diabetes had at least one of the 12 autoimmune diseases (27.03\%; 48,457/179,248; $95 \%$ $\mathrm{CI}=26.83 \%-27.24 \%$ ). The prevalence of autoimmune disease was 1.9 times greater in female than in male patients $(p<0.001)$. In female patients with type 1 diabetes, $35.62 \%$ had another autoimmune disease $(30,523 / 85,690 ; 95 \%$ $\mathrm{CI}=35.30 \%-35.94 \%)$ and, in male patients, $19.17 \%$ did $(17,934 / 93,558 ; 95 \% \mathrm{CI}=18.92 \%-19.42 \%)$. 
Table 1. Characteristics of Individuals with Type 1 Diabetes

\begin{tabular}{lccc}
\hline Characteristic & Female & Male & Total \\
\hline Individuals, $n$ (\%) & $85,690(47.8)$ & $93,558(52.2)$ & $179,248(100)$ \\
Age when enrolled, mean (SD) & $35.7(19.4)$ & $34.7(18.6)$ & $35.2(19.0)$ \\
Years enrolled, mean (SD) & $3.4(3.2)$ & $3.4(3.1)$ & $3.4(3.1)$ \\
Residence, $n$ (\%) & & & \\
New England, New Jersey & $4711(5.5)$ & $5156(5.5)$ & $9867(5.5)$ \\
New York, Pennsylvania & $3905(4.6)$ & $4393(4.7)$ & $8298(4.6)$ \\
Middle Atlantic & $8732(10.2)$ & $9409(10.1)$ & $18,141(10.1)$ \\
Southeast & $15,991(18.7)$ & $16,491(17.6)$ & $32,482(18.1)$ \\
Michigan, Indiana, Ohio, Kentucky & $9683(11.3)$ & $10,395(11.1)$ & $20,078(11.2)$ \\
North Central & $7524(8.8)$ & $8860(9.5)$ & $16,384(9.1)$ \\
Middle Central & $8324(9.7)$ & $9131(9.8)$ & $17,455(9.7)$ \\
South Central & $11,328(13.2)$ & $12,172(13.0)$ & $23,500(13.1)$ \\
Mountain & $8018(9.4)$ & $9060(9.7)$ & $17,078(9.5)$ \\
Pacific & $7474(8.7)$ & $8491(9.1)$ & $15,965(8.9)$ \\
Concomitant autoimmune disease, $n(\%)$ & & & \\
Rheumatoid arthritis & $2498(2.9)$ & $1012(1.1)$ & $3510(2.0)$ \\
Celiac disease & $1944(2.3)$ & $1328(1.4)$ & $3272(1.8)$ \\
Inflammatory bowel disease & $823(1.0)$ & $762(0.8)$ & $1585(0.9)$ \\
Pernicious anemia & $772(0.9)$ & $415(0.4)$ & $1187(0.7)$ \\
Hypothyroidism & $25,764(30.1)$ & $14,004(15.0)$ & $39,768(22.2)$ \\
Graves'disease & $1400(1.6)$ & $665(0.7)$ & $2065(1.2)$ \\
Addison's disease & $501(0.6)$ & $348(0.4)$ & $849(0.5)$ \\
Psoriasis & $1372(1.6)$ & $1191(1.3)$ & $2563(1.4)$ \\
Systemic lupus erythematosus & $584(0.7)$ & $113(0.1)$ & $697(0.4)$ \\
Multiple sclerosis & $455(0.5)$ & $200(0.2)$ & $655(0.4)$ \\
Sjogren's syndrome & $346(0.4)$ & $60(0.1)$ & $406(0.2)$ \\
Myasthenia gravis & $85(0.1)$ & $57(0.1)$ & $142(0.1)$ \\
\hline
\end{tabular}

$\mathrm{SD}$, standard deviation.

Of the 48,457 individuals with type 1 diabetes who had concomitant autoimmune diseases, $85.59 \%$ had only one concomitant disease $(n=41,476), 12.23 \%$ had two concomitant diseases $(n=5928), 1.84 \%$ had three $(n=891)$, and $0.33 \%(n=162)$ had four or more. The most common autoimmune diseases occurring together were hypothyroidism with rheumatoid arthritis.

\section{Age-specific prevalence of concomitant autoimmune disease in female and male patients}

Overall, there were considerable age and sex differences in the prevalence of concomitant autoimmune diseases in patients with type 1 diabetes. The pattern of occurrence varied for each of the diseases. Age- and sex-specific prevalence of concomitant autoimmune diseases for patients with type 1 diabetes are listed in Table 2 (females) and Table 3 (males). For example, a 45-year-old woman with type 1 diabetes has a $33 \%$ chance of having hypothyroidism, a $3 \%$ chance of having rheumatoid arthritis, and a $1 \%$ chance of having celiac disease, psoriasis, Graves' disease, inflammatory bowel disease, or pernicious anemia.

The overall prevalence of hypothyroidism in individuals with type 1 diabetes was $22.19 \%$ (95\% CI $=21.99 \%-$ $22.38 \%$ ). Diagnosis and treatment for hypothyroidism was most prevalent in middle-aged and older adults with type 1 diabetes. However, even in adolescents (ages 15-19 years), 1 in 7 female $(14.41 \%)$ and 1 in 14 male patients $(6.94 \%)$ had hypothyroidism. The prevalence was significantly greater $(p<0.001)$ in female $(30.07 \% ; 95 \% \mathrm{CI}=29.76 \%-30.37 \%)$ than in male patients $(14.97 \% ; 95 \% \mathrm{CI}=14.74 \%-15.20 \%)$.

The diagnosis of rheumatoid arthritis increased with age in patients with type 1 diabetes, until approximately 60-64 years of age and then decreased somewhat at the oldest age. The overall prevalence of rheumatoid arthritis in individuals with type 1 diabetes was $1.96 \%(95 \% \mathrm{CI}=1.89 \%-2.02 \%)$ and was significantly $(p<0.001)$ greater in female $(2.92 \%$; $95 \% \mathrm{CI}=2.80 \%-3.03 \%)$ than in male patients $(1.08 \% ; 95 \%$ $\mathrm{CI}=1.02 \%-1.15 \%)$.

Celiac disease occurred in $1.83 \%$ of persons with type 1 diabetes $(95 \% \mathrm{CI}=1.76 \%-1.89 \%)$. Diagnosis and treatment of celiac disease was greatest in children and adolescents 519 years of age and was quite low in older adults. Celiac disease occurred in $2.27 \%$ of female patients with type 1 diabetes $(95 \% \mathrm{CI}=2.17 \%-2.37 \%)$ and $1.42 \%$ of male patients with type 1 diabetes $(95 \% \quad \mathrm{CI}=1.34 \%-1.50 \%)$ $(p<0.001)$.

Adults with type 1 diabetes who were in their 50th and 60th decade of life were most likely to be diagnosed with psoriasis. Overall, $1.43 \%(95 \% \mathrm{CI}=1.38 \%-1.49 \%)$ of those with type 1 diabetes had psoriasis, with a greater prevalence in female $(1.60 \% ; 95 \% \mathrm{CI}=1.52 \%-1.69 \%)$ than in male patients $(1.27 \%$; $95 \% \mathrm{CI}=1.20 \%-1.35 \%)(p<0.001)$. The diagnosis of psoriasis was low at the very youngest age, increased somewhat in middle adulthood, and declined somewhat at the oldest age.

Graves' disease was most common in middle-aged adults with type 1 diabetes. Overall, Graves' disease occurred in $1.15 \%(95 \% \mathrm{CI}=1.10 \%-1.20 \%)$ of individuals with type 1 diabetes. The prevalence was significantly $(p<0.001)$ 


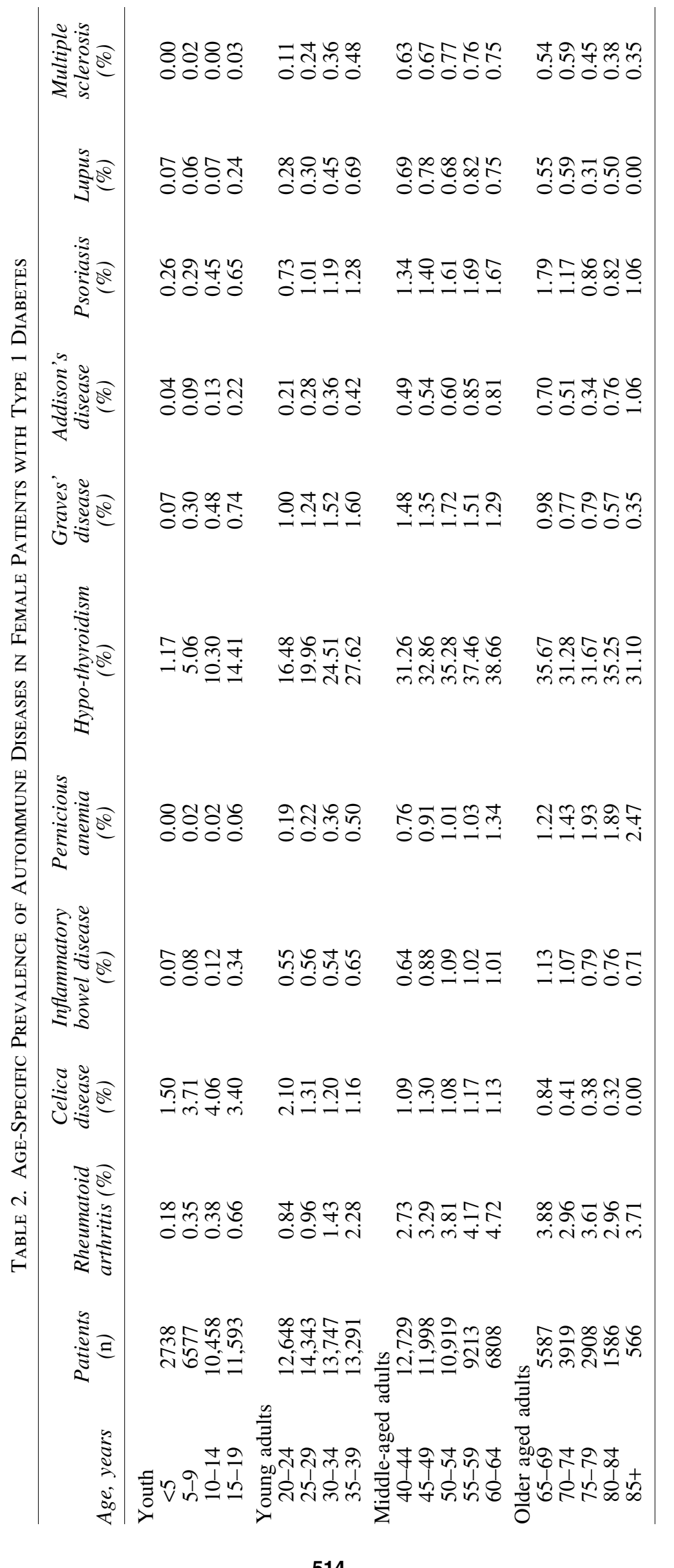




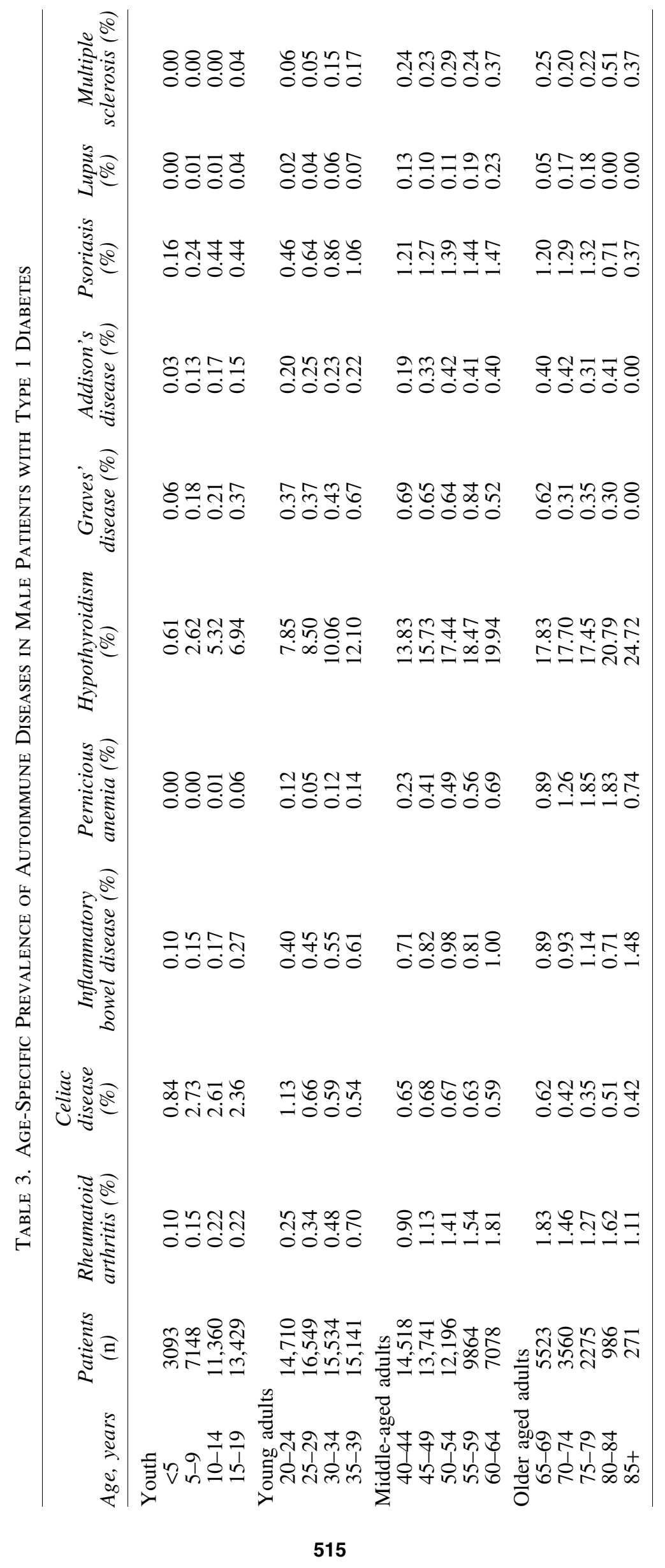


greater in female $(1.63 \% ; 95 \% \mathrm{CI}=1.55 \%-1.72 \%)$ than in male patients $(0.71 \% ; 95 \% \mathrm{CI}=0.66 \%-0.77 \%)$.

Diagnosis and treatment of inflammatory bowel disease occurred in $0.88 \%(95 \% \mathrm{CI}=0.84 \%-0.93 \%)$ of individuals with type 1 diabetes. It occurred most often in middle-aged and older adults. The prevalence was greater in female $(0.96 \% ; 95 \% \mathrm{CI}=0.90 \%-1.03 \%)$ than in male patients $(0.81 \% ; 95 \% \mathrm{CI}=0.76 \%-0.87 \%)(p=0.001)$, although difference was not as marked as in the other autoimmune disorders.

Pernicious anemia occurred in $0.90 \%$ of female patients with type 1 diabetes $(95 \% \mathrm{CI}=0.84 \%-0.97 \%)$ and $0.44 \%$ in male patients with type 1 diabetes $(95 \% \mathrm{CI}=0.40 \%-0.49 \%)$ $(p<0.001)$. Overall, the prevalence of pernicious anemia was $0.66 \%(95 \% \mathrm{CI}=0.63 \%-0.70 \%)$ and was greater in older adults.

The prevalence of Addison's disease was greater in adults with type 1 diabetes and overall, was $0.47 \% \quad(95 \%$ $\mathrm{CI}=0.44 \%-0.51 \%)$. Female patients were more likely to have Addison's disease $(0.58 \% ; 95 \% \mathrm{CI}=0.53 \%-0.64 \%)$ than male patients $(0.37 \% ; 95 \% \quad \mathrm{CI}=0.33 \%-0.41 \%)$ $(p<0.001)$.

Systemic lupus erythematosus occurred most often in middle-aged women with type 1 diabetes. The prevalence was lower in children and in the oldest adults. The prevalence of lupus was markedly greater in female $(0.68 \% ; 95 \%$ $\mathrm{CI}=0.63 \%-0.74 \%)$ than in male patients $(0.12 \% ; 95 \%$ $\mathrm{CI}=0.10 \%-0.15 \%)(p<0.001)$.

Multiple sclerosis occurred in $0.37 \%(95 \% \mathrm{CI}=0.34 \%-$ $0.39 \%$ ) of those with type 1 diabetes. Similar to lupus, it occurred more often in middle-aged women. The prevalence was $0.53 \%$ in female $(95 \% \mathrm{CI}=0.48 \%-0.58 \%)$ and $0.21 \%$ in male patients $(95 \% \mathrm{CI}=0.19 \%-0.25 \%)$ with type 1 diabetes $(p<0.001)$.

Sjögren's syndrome was principally a condition seen in women with type 1 diabetes; the prevalence was quite low in men. The prevalence of Sjögren's syndrome was $0.23 \%$ overall $(95 \% \mathrm{CI}=0.21 \%-0.25 \%)$, but was $0.40 \%(95 \%$ $\mathrm{CI}=0.36 \%-0.45 \%)$ in female and $0.06 \%(95 \% \mathrm{CI}=0.05 \%-$ $0.08 \%)$ in male patients $(p<0.001)$.

The prevalence of myasthenia gravis was quite low overall $(0.08 \%$; $95 \% \mathrm{CI}=0.07 \%-0.09 \%)$. The prevalence in female patients with type 1 diabetes was $0.10 \%(95 \% \mathrm{CI}=0.08 \%-$ $0.12 \%)$ and in male patients it was $0.06 \%(95 \% \mathrm{CI}=0.05 \%-$ $0.08 \%)(p=0.004)$.

\section{Association with renal and cardiovascular morbidities}

Renal failure occurred in 18,350 of the persons with type 1 diabetes and was more frequent in those with concomitant autoimmune disease $(15.1 \%$ with vs. $8.4 \%$ without; $p<0.001)$. Table 4 provides the ORs for the association between the number of concomitant autoimmune diseases and the outcomes (renal failure, ischemic stroke, myocardial infarction). As the number of additional diseases increased, so did the odds of renal failure $(p<0.001$, test for trend in both female and male patients). For those with four or more concomitant autoimmune diseases, the OR was 4.77 in female and 5.02 in male patients. The predicted probability of renal failure ranged from 0.12 in female patients with one concomitant autoimmune disease to 0.27 in female patients with four or more concomitant autoimmune diseases.

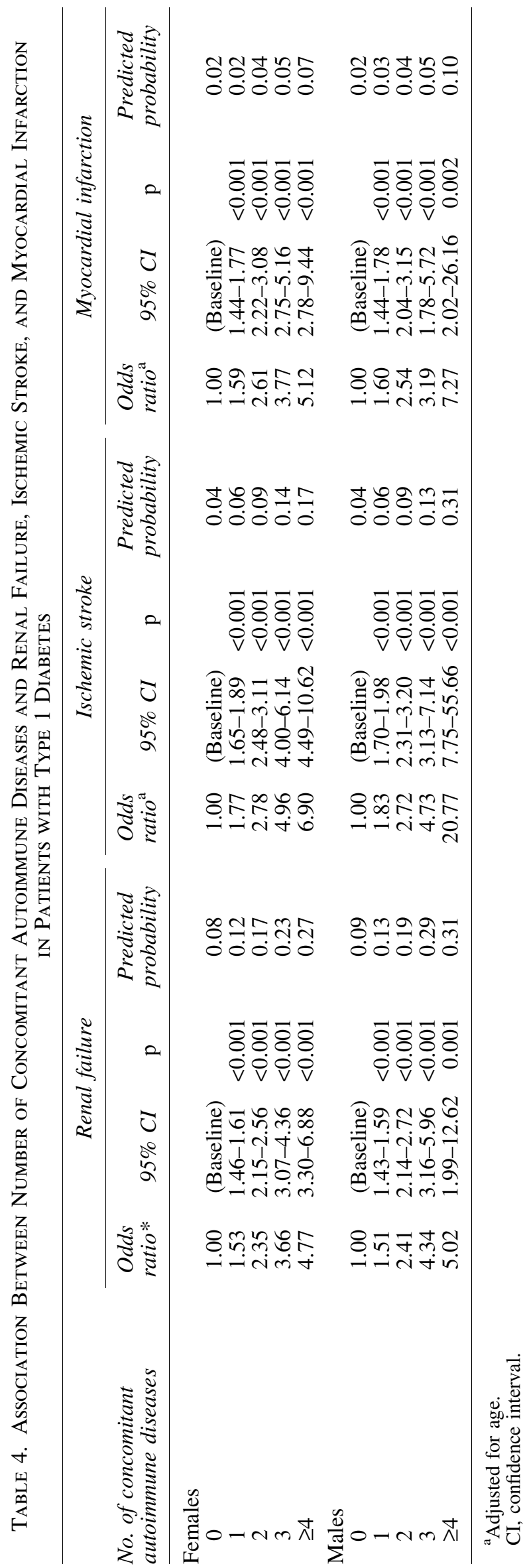


The corresponding probabilities for male patients were $0.13-$ 0.31 , respectively.

Ischemic stroke ( $n=8616)$ occurred in $8.3 \%$ of those with concomitant autoimmune disease, but in $3.5 \%$ of those with type 1 diabetes without another autoimmune disorder $(p<0.001)$. The odds of ischemic stroke increased with the number of concomitant autoimmune diseases $(p<0.001$, test for trend in both sexes). The predicted probability of ischemic stroke was 0.04 in patients with type 1 diabetes without concomitant autoimmune disease, but rose to 0.17 in female and 0.31 in male patients who had four or more concomitant autoimmune diseases (Table 4).

Myocardial infarction $(n=3711)$ was more likely to occur in patients with type 1 diabetes who had another autoimmune disease (3.4\%) compared with $1.6 \%$ in those without $(p<0.001)$. The ORs for the number of concomitant autoimmune diseases and myocardial infarction increased with the increasing number of concomitant autoimmune diseases $(p<0.001$, test for trend in both female and male patients). The predicted probabilities for myocardial infarction were lower than those for ischemic stroke in both sexes (Table 4).

There were no statistically significant interactions between sex and the number of concomitant autoimmune diseases for renal failure and myocardial infarction (interaction term, $p=0.277$ for renal failure, $p=0.205$ for myocardial infarction). However, the risk of ischemic stroke rose more dramatically in male patients as the number of concomitant autoimmune diseases increased compared with female patients ( $p=0.002$, interaction term), as given in Table 4 .

The association between specific concomitant autoimmune diseases and renal/cardiovascular morbidities are listed in Table 5. Individuals with type 1 diabetes who had Addison's disease were at considerably elevated risk of renal failure $(\mathrm{OR}=3.19)$, ischemic stroke $(\mathrm{OR}=2.24)$, and myocardial infarction $(\mathrm{OR}=2.34)$. Similarly, concomitant systemic lupus erythematosus was associated with approximately twofold greater risk of renal failure, ischemic stroke, and myocardial infarction. Individuals with type 1 diabetes and myasthenia gravis were at particularly greater risk of ischemic stroke $(\mathrm{OR}=3.22)$, as were patients with multiple sclerosis $(\mathrm{OR}=3.57)$.
Although renal and cardiovascular morbidities were less common in children than adults, there were some children and adolescents with these outcomes. The OR for renal disease, comparing individuals with and without concomitant autoimmune disease, was $1.85(95 \% \mathrm{CI}=1.62-2.10)$ in patients who were $<20$ years of age. For ischemic stroke, the corresponding OR was $2.11(95 \% \mathrm{CI}=1.44-3.10)$. For myocardial infarction, there was no association in patients $<20$ years ( $p=0.134$ comparing with and without concomitant autoimmune disease).

\section{Discussion}

Multimorbidity of autoimmune disease is common in women with type 1 diabetes-occurring in one in three patients-and is associated with substantially greater frequencies of renal failure, ischemic stroke, and myocardial infarction. Concomitant autoimmune disease in male patients with type 1 diabetes was less frequent, occurring in one in five male patients, but also was strongly associated with renal and cardiovascular outcomes. The greater the number of autoimmune diseases, the greater the risk. Patients with type 1 diabetes who also had Addison's disease, systemic lupus erythematosus, multiple sclerosis, or myasthenia gravis were at particular risk.

Studies using large patient registries also found a high prevalence of concomitant autoimmune diseases in persons with type 1 diabetes, although there is some variation in prevalence across studies. ${ }^{6,7}$ In a large study of autoimmune disease in adults with type 1 diabetes, one in five patients with type 1 diabetes had thyroid disease, although the authors used a broader definition of thyroid disease that combined hypothyroidism and hyperthyroidism of unspecified etiologies. ${ }^{7}$ Our study differs in that we included both children and adults. Our findings demonstrate that certain autoimmune conditions occur more often in children with type 1 diabetes, such as celiac disease, but others such as pernicious anemia are more frequent in older adults.

Our study highlights the importance of taking a comprehensive history of autoimmune disorders, particularly in

Table 5. Association Between Specific Concomitant Autoimmune Diseases and Renal Failure, Ischemic Stroke and Myocardial Infarction in Patients with Type 1 Diabetes

\begin{tabular}{|c|c|c|c|c|c|c|}
\hline \multirow{2}{*}{$\begin{array}{l}\text { Concomitant autoimmune } \\
\text { disease }\end{array}$} & \multicolumn{2}{|c|}{ Renal failure } & \multicolumn{2}{|c|}{ Ischemic stroke } & \multicolumn{2}{|c|}{ Myocardial infarction } \\
\hline & Odds ratio ${ }^{\mathrm{a}}$ & $95 \% C I$ & Odds ratio $^{\mathrm{a}}$ & $95 \% C I$ & Odds ratio $^{\mathrm{a}}$ & $95 \% C I$ \\
\hline Rheumatoid arthritis & 1.47 & $1.35-1.61$ & 1.79 & $1.60-1.99$ & 1.68 & $1.43-1.96$ \\
\hline Celiac disease & 1.16 & $1.02-1.33$ & 1.28 & $1.04-1.56$ & 1.33 & $1.01-1$. \\
\hline Inflammatory bowel disease & 2.17 & $1.92-2.45$ & 1.82 & $1.54-2.16$ & 1.34 & $1.03-1.74$ \\
\hline Pernicious anemia & 2.16 & $1.89-2.46$ & 2.14 & $1.82-2.50$ & 1.99 & $1.61-2.47$ \\
\hline Hypothyroidism & 1.38 & $1.33-1.43$ & 1.63 & $1.55-1.71$ & 1.46 & $1.36-1.5$ \\
\hline Graves' disease & 1.04 & $0.91-1.20$ & 1.16 & $0.96-1.40$ & 1.44 & $1.12-1.8$ \\
\hline Addison's disease & 3.19 & $2.73-3.73$ & 2.24 & $1.80-2.77$ & 2.34 & $1.76-3$. \\
\hline Psoriasis & 1.19 & $1.06-1.33$ & 1.26 & $1.08-1.48$ & 1.28 & $1.03-1.60$ \\
\hline Systemic lupus erythematosus & 2.55 & $2.14-3.04$ & 2.15 & $1.71-2.71$ & 1.97 & $1.42-2.73$ \\
\hline Multiple sclerosis & 1.92 & $1.59-2.32$ & 3.57 & $2.88-4.42$ & 1.91 & $1.35-2.69$ \\
\hline Sjogren's syndrome & 1.25 & $0.98-1.60$ & 1.58 & $1.18-2.11$ & 0.71 & $0.42-1.20$ \\
\hline Myasthenia gravis & 1.85 & $1.26-2.73$ & 3.22 & $2.11-4.91$ & 2.07 & $1.14-3.76$ \\
\hline
\end{tabular}

${ }^{\mathrm{a}}$ Adjusted for age. 
women with type 1 diabetes. All 12 concomitant autoimmune diseases occurred more frequently in female patients, although there was variation in the age in which specific diseases occurred. Other investigators have also found a greater prevalence of concomitant autoimmune diseases in female with type 1 diabetes compared with male patients. ${ }^{6,7} \mathrm{We}$ found that the degree of the female predominance depends upon the age of the patient and the specific type of concomitant disease.

At present, the components of the comprehensive diabetes medical evaluation of patients with type 1 diabetes indicate that the presence of autoimmune diseases be ascertained at medical visits. ${ }^{17}$ For laboratory evaluation, screening for autoimmune thyroid disease and celiac disease are recommended soon after the diagnosis of type 1 diabetes. ${ }^{17}$ Our findings underscore the importance of these recommendations and suggest that this evaluation is especially pertinent to girls and women with type 1 diabetes, who are at greater risk for all of the concomitant autoimmune diseases studied here.

Of importance, concomitant autoimmune diseases were associated with renal failure, ischemic stroke, and myocardial infarction. With each additional autoimmune disease, the likelihood of renal failure, stroke, and myocardial infarction increased. In previous research, there was a fourfold increased risk of death in those with both diabetes and Addison's disease, usually from cardiovascular disease. ${ }^{18}$ Coronary heart disease including myocardial infarction occurred in $7.4 \%$ of those with both type 1 diabetes and Addison's disease, whereas it in $4.9 \%$ in those with type 1 diabetes only. ${ }^{18}$ In a different investigation, the incidence of myocardial infarction was 13.0 per 1000 person-years in those with both diabetes and rheumatoid arthritis, which was higher than in those with diabetes alone $(8.1 / 1000$ personyears) or in those with rheumatoid arthritis alone $(5.7 / 1000$ person-years). ${ }^{19}$

It is unknown whether the risk of renal and cardiovascular disease in persons with multiple autoimmune disease is because of the complexities of underlying pathophysiology or whether subsequent chronic conditions render management of type 1 diabetes more difficult. Additional information is necessary to determine whether there are particular aspects of diabetes management that are challenging given the presence of another condition. Previous research has shown that in some instances (e.g., type 1 diabetes with celiac disease), treatment of celiac disease with a gluten-free diet decreased hypoglycemic episodes but then increased the need for larger doses of insulin. ${ }^{20,21}$ Other studies indicate that treatment for the autoimmune disorder (e.g., Addison's disease, rheumatoid arthritis) with corticosteroids may result in hyperglycemia. ${ }^{22}$ This can pose challenges to the daily management of glucose levels and may impact long-term risk of diabetic complications.

There are limitations of this study. Information regarding $\mathrm{HbA} 1 \mathrm{c}$ and duration of type 1 diabetes were only available on a subset of individuals and therefore, are not included in this study. In addition, we did not capture all autoimmune diseases among persons with type 1 diabetes; we concentrated on the 12 most frequent conditions in this population. In patients with type 1 diabetes, there also may be other nonautoimmune-related comorbidities beyond those studied here. Therefore, it is likely that the overall burden of multimorbidity may be greater. However, the conditions captured here are among those associated with the worst physical health-related quality of life and mortality risk. ${ }^{2}$

Strengths of this study include the precision of the estimates owing to the large sample size and the inclusion of individuals of all ages. Because of this, we incorporated data on patients with several rare chronic conditions such as multiple sclerosis. In previous studies, autoimmune conditions such as multiple sclerosis had devastating effects on physical health-related quality of life. ${ }^{2}$ The prevalence and prognosis of such rare conditions are often underestimated because large national studies in the United States do not obtain these data. In addition, the inclusion of all age groups adds to the body of literature on multimorbidity because most published studies are of older adults - not fully capturing the impact of chronic conditions that originate earlier in life. Although renal/cardiovascular outcomes are rarer in young patients, children with type 1 diabetes and concomitant autoimmune disease were at increased risk of renal failure and ischemic stroke.

In conclusion, we found that the presence of concomitant autoimmune disease in patients with type 1 diabetes was common, occurring in one of every three women and one of every five men. Multimorbidity in patients with type 1 diabetes was strongly associated with renal failure, ischemic stroke, and myocardial infarction. Because concomitant autoimmune disease is more frequent in women than men with type 1 diabetes, these renal and cardiovascular diseases affect greater numbers of women. We recommend periodic screening for autoimmune diseases throughout the lifespan of patients with type 1 diabetes. We encourage initiatives to evaluate the effectiveness of more intensive preventive treatments to decrease the incidence of renal failure, ischemic stroke, and myocardial infarction in patients with type $1 \mathrm{di}-$ abetes.

\section{Authors' Contributions}

All authors contributed to the concept and design. M.A.M.R. and J.M.L. acquired the data. M.A.M.R. analyzed the data. All authors participated in drafting the article or revising it critically for intellectual content. All authors approved of the final version.

\section{Author Disclosure Statement}

No competing financial interests exist.

\section{Funding Information}

This study was funded by the National Institutes of Health (Grant UL1TR000433) to the Michigan Institute for Clinical $\&$ Health Research and by the Jaeb Center for Health Research Foundation, Inc. (T1D Registry). M.Y.W. is funded by the National Institute on Aging (K23AG056638). The funders had no role in the design of the study, or the collection, analysis and interpretation of data, or in writing the article.

\section{References}

1. Ward BW, Schiller JS, Goodman RA. Multiple chronic conditions among US adults: A 2012 update. Prev Chronic Dis 2014;11:E62.

2. Wei MY, Mukamal KJ. Multimorbidity, mortality and long-term physical functioning in three prospective cohorts 
of community-dwelling adults. Am J Epidemiol 2018;187: 103-112.

3. Wei MY, Mukamal KJ. Multimorbidity and mental healthrelated quality of life and completed suicide. J Am Geriatr Soc 2019;67:511-519.

4. Wei MY, Kawachi I, Okereke OI, Mukamal KJ. Diverse cumulative impact of chronic diseases on physical healthrelated quality of life: Implications for a measure of multimorbidity. Am J Epidemiol 2016;184:357-365.

5. Rogers MA, Kim C, Banerjee T, Lee JM. Fluctuations in the incidence of type 1 diabetes in the United States from 2001 to 2015: A longitudinal study. BMC Med 2017;15: 199.

6. Hughes JW, Riddlesworth TD, DiMeglio LA, et al. Autoimmune diseases in children and adults with type 1 diabetes from the T1D exchange clinic registry. J Clin Endocrinol Metabo 2016;101:4931-4937.

7. Bao YK, Weide LG, Ganesan VC, et al. High prevalence of comorbid autoimmune diseases in adults with type 1 diabetes from the HealthFacts database. J Diabetes 2019;11: 273-279.

8. Buschur E, Sarma AV, Pietropaolo M, et al. Self-reported autoimmune disease by sex in the Diabetes Control and Complications Trial/Epidemiology of Diabetes Interventions and Complications (DCCT/EDIC) Study. Diabetes Care 2014;37:e28-e29.

9. Triolo TM, Armstrong TK, McFann K, et al. Additional autoimmune disease found in $33 \%$ of patients at type 1 diabetes onset. Diabetes Care 2011;34:1211-1213.

10. Atkinson MA, Eisenbarth GS, Michels AW. Type 1 diabetes. Lancet 2014;383:69-82.

11. Adamsson Eryd S, Svensson AM, Franzén S, Eliasson B, Nilsson PM, Gudbjörnsdottir S. Risk of future microvascular and macrovascular disease in people with Type 1 diabetes of very long duration: A national study with 10year follow-up. Diabet Med 2017;34:411-418.

12. Patterson C, Guariguata L, Dahlquist G, Soltész G, Ogle G, Silink M. Diabetes in the young-A global view and worldwide estimates of numbers of children with type 1 diabetes. Diabetes Res Clin Pract 2014;103:161-175.

13. Diaz-Valencia PA, Bougnères $P$, Valleron AJ. Global epidemiology of type 1 diabetes in young adults and adults: A systematic review. BMC Public Health 2015;15:255.

14. Zhong VW, Pfaff ER, Beavers DP, et al. Use of administrative and electronic health record data for development of automated algorithms for childhood diabetes case ascertainment and type classification: The SEARCH for diabetes in youth study. Pediatr Diabetes 2014;15:573-584.

15. Klompas M, Eggleston E, McVetta J, Lazarus R, Li L, Platt R. Automated detection and classification of type 1 versus type 2 diabetes using electronic health record data. Diabetes Care 2013;36:914-921.

16. American Diabetes Association. Economic costs of diabetes in the US in 2017. Diabetes Care 2018;41:917.

17. American Diabetes Association. 4. Comprehensive medical evaluation and assessment of comorbidities: Standards of medical care in diabetes-2019. Diabetes Care 2019; 42(Suppl 1):S34-S45.

18. Chantzichristos D, Persson A, Eliasson B, et al. Mortality in patients with diabetes mellitus and Addison's disease: A nationwide, matched, observational cohort study. Eur J Endocrinol 2017;176:31-39.

19. Lindhardsen J, Ahlehoff O, Gislason GH, et al. The risk of myocardial infarction in rheumatoid arthritis and diabetes mellitus: A Danish nationwide cohort study. Ann Rheum Dis 2011;70:929-934.

20. Abid N, McGlone O, Cardwell C, McCallion W, Carson D. Clinical and metabolic effects of gluten free diet in children with type 1 diabetes and coeliac disease. Ped Diabetes 2011;12(4pt1):322-325.

21. Taler I, Phillip M, Lebenthal Y, de Vries L, Shamir R, Shalitin S. Growth and metabolic control in patients with type 1 diabetes and celiac disease: A longitudinal observational case-control study. Ped Diabetes 2012;13:597606.

22. Varas-Lorenzo C, Rodriguez LA, Maguire A, Castellsague J, Perez-Gutthann S. Use of oral corticosteroids and the risk of acute myocardial infarction. Atherosclerosis 2007;192: 376-383.

Address correspondence to: Mary A.M. Rogers, PhD Department of Internal Medicine University of Michigan

Building 16, Room 422W North Campus Research Complex, 2800 Plymouth Road Ann Arbor, MI 48109-2800

E-mail: maryroge@umich.edu 